\title{
Enhanced magnetic properties of FeCo ribbons nanocrystallized in magnetic field
}

\author{
P. Marín ${ }^{\text {a) }}$ and A. Hernando \\ Departamento de Física de Materiales, Instituto de Magnetismo Aplicado, Universidad Complutense de \\ Madrid-ADIF-CSIC, P.O. Box 155, Las Rozas, 28230 Madrid, Spain
}

(Received 13 January 2009; accepted 26 January 2009; published online 25 March 2009)

\begin{abstract}
Tailoring the structure of nanocrystalline microstructures is an important step toward controlled design of novel nanostructured materials and devices. We demonstrate how the nanocrystalline microstructure of Co-rich ribbons can be tuned by annealing under magnetic field. The intensity of the field allows controlling different degrees of order at annealing temperatures corresponding to the first stages of the nanocrystallization process. The energy barrier for nucleation is directionally affected by the applied field. The influence of grains assembling on exchange coupling between grains has been analyzed by means of magnetic domains observation and magnetic characterization by means of a hysteresis loop. (C) 2009 American Institute of Physics. [DOI: 10.1063/1.3091401]
\end{abstract}

The assembly of nanoparticles in ordered structures is observed in materials of biological, geological, and manmade origin. ${ }^{1-3}$ It has been found in magnetic nanoparticles buried in a dielectric nonmagnetic metal matrix, ${ }^{4}$ polymer mediated, ${ }^{5}$ or with biomolecules synthesized in an aqueous solution. ${ }^{6}$ Promising attempts of applying a magnetic field as an external agent to influence in nanoparticles organization has been done. Sui et al. ${ }^{7}$ assembled FePt $L 1_{0}$-ordered clusters through the use of ordered nanopore templates and external magnetic field. Ahniyaz et al. ${ }^{8}$ showed how the application of an external magnetic field on a dispersion of superparamagnetic maghmetite nanocubes can induce the formation of defect-free ordered superlattices. When magnetic field is applied during evaporation under air, linear structures of cobalt nanocrystals are observed. ${ }^{9}$

First iron based nanocrystalline alloys presenting superior soft magnetic behavior were produced by partial crystallization of an amorphous $\mathrm{Fe}-\mathrm{Si}-\mathrm{B}-\mathrm{Cu}-\mathrm{Nb}$ alloy. ${ }^{10}$ Softness mainly arises from the averaging out of magnetocrystalline anisotropy. ${ }^{11}$ The control of amorphous percentage and grain size should be done through composition and annealing temperature. $^{12}$

In previous works, ${ }^{13}$ we presented the possibility of tuning the structure of Co-rich nanograins by means of magnetic field annealing. We observed a noticeable influence of magnetic field on the nanocrystallization of $\mathrm{Fe}_{28.5} \mathrm{Co}_{45} \mathrm{Si}_{13.5} \mathrm{~B}_{9} \mathrm{Cu}_{1} \mathrm{Nb}_{3}$ (FeCo-rich alloys). An applied field during the first stage of nanocrystallization, at $733 \mathrm{~K}$, exerts a drastic influence on both percentage of crystallized material and size of the precipitated fcc $\mathrm{FeCoSi}$ grains. Moreover, the effect of the applied field is also reflected in the assembly of nanograins that show aligned patterns as well as crystalline texture on [1ㅣㅣㄹ direction, confirmed by Fourier Transform (FT) along [110] and by x-ray diffraction. ${ }^{13}$ The directional order induced by the field is characterized by a decrease in the intergrain distance along the field direction when compared to the perpendicular one.

\footnotetext{
a) Author to whom correspondence should be addressed. Tel.: +34913007 173. FAX: +34913007 176. Electronic addresses: pmarin@adif.es and pmarin@renfe.es.
}

The energy barrier for nucleation is directionally affected by the applied field.

Other authors have analyzed the influence of nanocrystalline microstructure on magnetic domains. ${ }^{14-18}$ Domains patterns depend on the competition between random and uniform anisotropy. ${ }^{15}$ The aim of the present work is to analyze the influence of custom assembly of nanograins in the collective magnetic behavior of the samples by means of the hysteresis loop and magnetic domains character.

A rapidly solidified $\mathrm{Fe}_{28.5} \mathrm{Co}_{4} 5 \mathrm{Si}_{13.5} \mathrm{Cu}_{1} \mathrm{Nb}_{3}$ alloy was annealed for $1 \mathrm{~h}$ between 573 and $823 \mathrm{~K}$ in argon atmosphere under magnetic field applied along the ribbon longitudinal direction (ribbon axis), between 800 and $11.200 \mathrm{~A} \mathrm{~m}^{-1}$. The hysteresis loops were obtained by a conventional $80 \mathrm{~Hz}$ induction technique, while saturation magnetization was determined by a vibrating sample magnetometry. The nanocrystalline microstructure was confirmed and analyzed by transmission electron microscopy (JEOL JEM 3000 FX). The domain structure was observed by Kerr microscopy in a standard optical wide-field polarization microscope. The axis of the magneto-optical sensitivity is vertical for all images.

Figure 1 shows transmission electron microscopy (TEM) micrographs of the alloy annealed at $733 \mathrm{~K}$ under $0,4.000$, and $11.200 \mathrm{~A} \mathrm{~m}^{-1}$ and at $793 \mathrm{~K}$ without magnetic field. $\mathrm{Ob}$ viously, from Fig. 1, the crystallization process has been modified by the effect of magnetic field. Figure 1(a) shows the microstructure corresponding to the sample annealed without magnetic field consisting in a random distribution of grains of medium size of $6 \mathrm{~nm}$ surrounded by an amorphous matrix. In the case of $4.000 \mathrm{~A} \mathrm{~m}^{-1}$ field [Fig. 1(b)], a grains self-assembly along field direction (ribbon axis) is observed. This effect is associated to an increase in grain size to $10 \mathrm{~nm}$. The effect at $11.200 \mathrm{~A} \mathrm{~m}^{-1}$ is shown on Fig. 1(c) where a coexistence of $25 \mathrm{~nm}$ nanocrystalline grains embedded in a less amount of amorphous phase is observed. A higher percentage of nanocrystallized material with random FeCo grain distribution is shown for sample annealed at $793 \mathrm{~K} .{ }^{13}$

The corresponding hysteresis loops for the microstructures shown in Fig. 1 can be seen in Fig. 2(a). If we compare the hysteresis loops of samples annealed at 733 [Fig. 2(a)1] and $793 \mathrm{~K}$ [Fig. 2(a)4] without magnetic field we observe a 


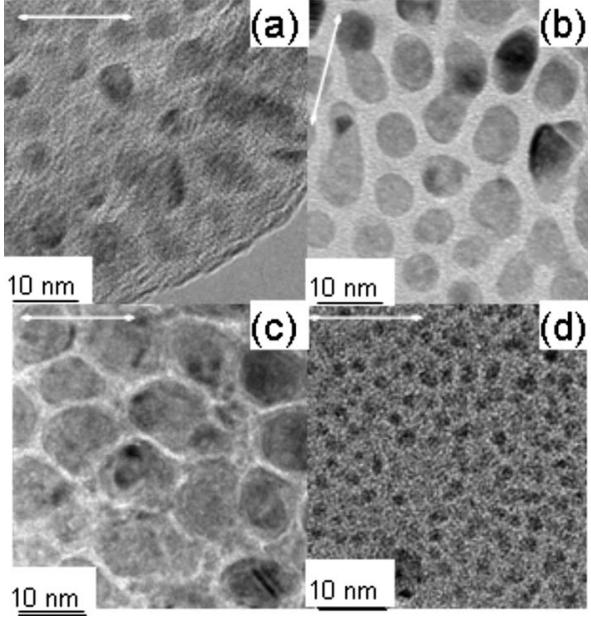

FIG. 1. TEM image for annealed FeCoSiBCuNb sample under magnetic field: (a) $733 \mathrm{~K}, H=0 \mathrm{~A} \mathrm{~m}^{-1}$; (b) $733 \mathrm{~K}, H=4.000 \mathrm{~A} \mathrm{~m}^{-1}$; (c) $733 \mathrm{~K}$, $H=11.200 \mathrm{~A} \mathrm{~m}^{-1}$; and (d) $793 \mathrm{~K}, H=0 \mathrm{~A} \mathrm{~m}^{-1}$. Magnetic field direction $(\bullet-\downarrow)$ is indicated.

softer magnetic behavior for that annealed at the lower temperature. A clear change in the hysteresis loop shape, associated to an increase in perpendicular magnetic anisotropy, is obtained for samples annealed at $793 \mathrm{~K}$. This change on magnetic behavior should be related to the differences observed on microstructure. In the first case, although the percentage of nanocrystalline phase is smaller than in the second one, the magnetocrystalline anisotropy of the Fe based grains is small enough to give an exchange length higher than the grain size and the distance between the grains. The modified random anisotropy model that considers the influence of the amorphous phase on the exchange coupling of the nanocrystals could be applied to calculate the coercivity field. ${ }^{11}$ In the case of the sample annealed at $793 \mathrm{~K}$, the cobalt presence in the grains should be the responsible of the harder magnetic behavior.

The influence of the magnetic field strength during annealing has been analyzed for the samples annealed at $733 \mathrm{~K}$. As it can be observed in the hysteresis loops for samples
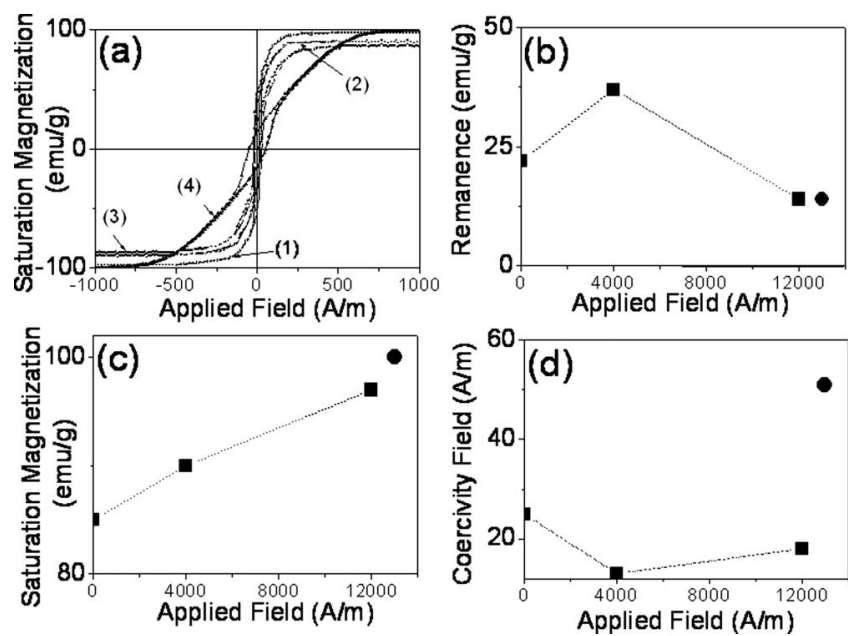

FIG. 2. (a) Influence on hysteresis loop of magnetic field intensity during annealing of $\mathrm{Fe}_{28.5} \mathrm{Co}_{45} \mathrm{Si}_{13.5} \mathrm{~B}_{9} \mathrm{Cu}_{1} \mathrm{Nb}_{3}$ ribbons at $733 \mathrm{~K}: 11.200 \mathrm{~A} \mathrm{~m}^{-1}$ (1), $4.000 \mathrm{~A} \mathrm{~m}^{-1}(2), 0 \mathrm{~A} \mathrm{~m}^{-1}(3)$, and $793 \mathrm{~K}$ (4) and influence of magnetic field intensity during annealing at $733 \mathrm{~K}$ on remanence (b), saturation magnetization (c), and coercivity (d). The corresponding data for the sample treated at $793 \mathrm{~K}$. without magnetic field has been also includeci (a)
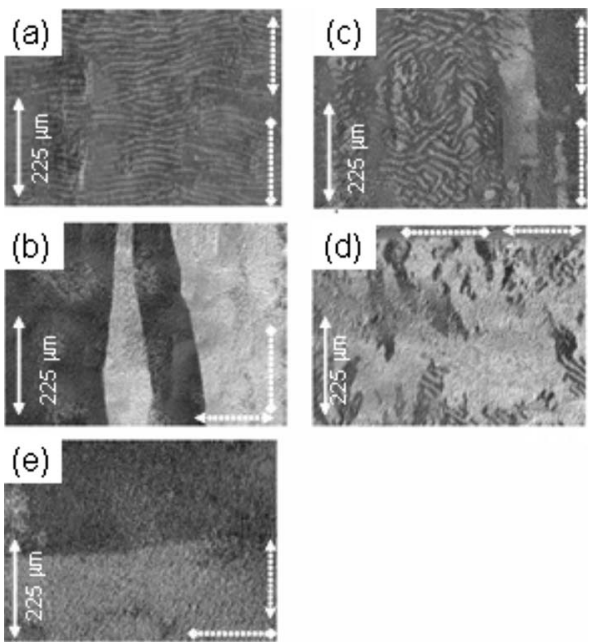

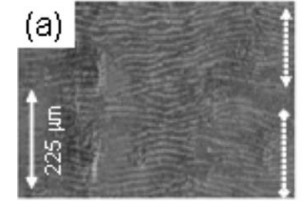

FIG. 3. Magnetic domains for annealed $\mathrm{FeCoSiBCuNb}$ sample under magnetic field: (a) $733 \mathrm{~K}, H=0 \mathrm{~A} \mathrm{~m}^{-1}$; (b) $733 \mathrm{~K}, H=4.000 \mathrm{~A} \mathrm{~m}^{-1}$; (c) $733 \mathrm{~K}$, $H=11.200 \mathrm{~A} \mathrm{~m}^{-1}$, and (d) $793 \mathrm{~K}, H=0 \mathrm{~m}^{-1}$. Magnetic field direction $(\bullet-\bullet)$ and ribbon axis $(\leftrightarrow)$ are indicated.

annealed under 4.000 [Fig. 2(a)2] and $11.200 \mathrm{~A} \mathrm{~m}^{-1}$ [Fig. 2(a)1], a longitudinal magnetic anisotropy is induced.

Figure 2 also shows the evolution of remanence (b), saturation magnetization (c), and coercivity (d) with the intensity of the applied field for sample annealed at $733 \mathrm{~K}$. In the three cases, the values are shown in comparison to that of the sample annealed at $793 \mathrm{~K}$ in the absence of applied magnetic field. The coercivity field decrease associated to an increase in remanence observed in the sample annealed under $4.000 \mathrm{~A} / \mathrm{m}$ should be related with the custom assembly of the grains. The saturation magnetization increase confirms the raise of nanocrystalline percentage with magnetic field intensity.

All these different microstructures are responsible for magnetic domain configurations. Figure 3 shows magnetic domain observation for samples annealed at $733 \mathrm{~K}$ under magnetic field [Figs. 3(b), 3(c), and 3(e)] and at $793 \mathrm{~K}$ without magnetic field [Fig. 3(d)]. In the case of sample annealed without magnetic field [Fig. 3(a)], the magnetic microstructure is inhomogeneous and dominated by stripelike stress domains. The two images shown are taken in the demagnetized state. In the case of the sample annealed at $733 \mathrm{~K}$ under 4.000 $\mathrm{A} \mathrm{m}^{-1}$ [Figs. 3(b) and 3(e)], wide longitudinal domains are visible in the demagnetized state. Stripelike stress domains and wide domains in the longitudinal direction are visible for the sample annealed at $733 \mathrm{~K}$ under 11.200 $\mathrm{A} \mathrm{m}^{-1}$ [Fig. 3(c)]. In the last case, sample annealed at $793 \mathrm{~K}$ [Fig. 3(d)], immobile domains dominate the magnetic microstructure. The magnetization process is governed by domain nucleation.

The influence of tuned microstructures obtained by means of annealing temperature and magnetic field intensity on sample magnetic behavior is shown here. The used composition $\mathrm{Fe}_{28.5} \mathrm{Co}_{45} \mathrm{Si}_{13.5} \mathrm{~B}_{9} \mathrm{Cu}_{1} \mathrm{Nb}_{3}$, allows to obtain a sample partially nanocrystalline with grains of size around $5 \mathrm{~nm}$ randomly distributed with lower coercivity and higher longitudinal anisotropy than the corresponding amorphous phase. It is also possible to obtain a random grain distribution with lower percentage of amorphous phase and grains slightly bigger when annealing between 823 and $793 \mathrm{~K}$. In this case, the hysteresis loops reveal an increase in perpendicular an- 
isotropy that should be related with an increase in cobalt percentage in sample nanograins. ${ }^{2}$ An alignment of grains is observed when annealing under magnetic field at $733 \mathrm{~K}$. The custom assembly of grains is related with coercivity decrease, as shown in Fig. 2.

In the case of soft magnetic nanocrystalline materials, ${ }^{15}$ the microstructure leads to a distribution of magnetic anisotropy axes randomly varying their orientation over the scale of the grain size $D$. As $D$ is smaller than the ferromagnetic correlation length $L_{\mathrm{ex}}$, the smoothing action of exchange energy impedes the magnetization to follow the local anisotropy axes. This would be the origin of a homogeneous domain structure like the one observed on sample annealed at $733 \mathrm{~K}$ under $4.000 \mathrm{~A} \mathrm{~m}^{-1}$. The wide and longitudinal domains observed should be a consequence of the random and uniform (induced) anisotropy. The observed regular domains should be a consequence of magnetostriction averaged out.

The observed domains on Fig. 3(a) look like as if the patterns have been "frozen-in" according to a stressdominated domain configuration present during annealing with nonzero magnetostriction constant.

Figure 3(c) shows the magnetic domains corresponding to the microstructure shown in Fig. 1(c). These grains are considerably larger than in Figs. 1(a) and 1(b). This might by contributing to the fact that the domains are getting complicated.

According to random anisotropy model, ${ }^{1}$ the effective anisotropy constant $\langle K\rangle$ is equal to $K_{1} / \sqrt{N}$, where $K_{1}$ is the magnetocrystalline anisotropy and $N$ is the number of exchange coupled grains within the renormalized correlation volume. Consequently the magnetic microstructure of nanocrystalline ribbons can be modified by changing $K_{1}$. This happens in Fig. 3(d), where an increase in $K_{1}$ due to cobalt diffusion makes random anisotropy less effectively averaged out and a patchy and immobile domains structure is observed. A similar magnetization process has been observed for the Finemet type nanocrystalline ribbons with transverse induced anisotropy above the Curie temperature of the amorphous phase (reduced exchange coupling). ${ }^{15,16}$
The possibility of tuning the nanostructure of magnetic materials by means of annealing under magnetic field has been confirmed by means of hysteresis loops and magnetic domains observations. The grains alignment observed is associated with a decrease in coercivity and wide longitudinal magnetic domains in the demagnetized state.

This work has been supported by the MEC of Spain (Consolider-Ingenio 2010 Contract No. CSD2007-0010, Nanociencia Molecular). The authors want to acknowledge the contribution of Professor J. M. González-Calbet and Dr. M. L. Ruiz-González in the HREM studies.

${ }^{1}$ H. Cölfen and S. Mann, Angew. Chem., Int. Ed. 42, 2350 (2003).

${ }^{2}$ M. Jose-Yacaman, L. Rendon, J. Arenas, and M. C. S. Puche, Science 273, 223 (1996).

${ }^{3}$ J. H. L. Watson, R. R. Cardell, and W. Heller, J. Phys. Chem. 66, 1757 (1962).

${ }^{4}$ C. L. Chien, J. Appl. Phys. 69, 5267 (1991).

${ }^{5}$ S. Sun, S. Anders, H. F. Hamann, J. U. Thiele, J. E. E. Baglin, T. Thomson, E. E. Fullerton, C. B. Murray, and B. D. Terris, J. Am. Chem. Soc. 124, 2884 (2002).

${ }^{6}$ T. Kim, L. Reis, K. Rajan, and M. Shima, J. Magn. Magn. Mater. 295, 132 (2005).

${ }^{7}$ Y. C. Sui, W. Liu, L. P. Yue, X. Z. Li, J. Zhou, R. Skomski, and D. J. Sellmyer, J. Appl. Phys. 97, 10J304 (2005).

${ }^{8}$ A. Ahniyaz, Y. Sakamoto, and L. Bergstrom, Proc. Natl. Acad. Sci. U.S.A. 104, 17570 (2007).

${ }^{9}$ M. P. Pileni, Pure Appl. Chem. 74, 1707 (2002).

${ }^{10}$ K. Suzuki, A. Makino, N. Kataoka, A. Inoue, and T. Masumoto, Mater. Trans., JIM 32, 93 (1991).

${ }^{11}$ A. Hernando, M. Vázquez, T. Kulik, and C. Prados, Phys. Rev. B 51, 3581 (1995)

${ }^{12}$ M. Vázquez, P. Marín, H. A. Davies, and A. O. Olofinjana, Appl. Phys. Lett. 64, 3184 (1994)

${ }^{13}$ P. Marín, M. López, A. Vlad, A. Hernando, M. L. Ruiz-González, and J. M. González-Calbet, Appl. Phys. Lett. 89, 033508 (2006).

${ }^{14}$ R. Schafer, A. Hubert, and G. Herzer, J. Appl. Phys. 69, 5325 (1991).

${ }^{15}$ S. Flohrer, R. Schafer, C. Polak, G. Herzer, and L. Schultz, Acta Mater. 53, 2937 (2005).

${ }^{16}$ S. Flohrer, R. Schäfer, J. McCord, S. Roth, G. Herzer, and L. Schultz, Acta Mater. 54, 3253 (2006).

${ }^{17}$ D. Shindo, Y.-G. Park, and Y. Yoshizawa, J. Magn. Magn. Mater. 238, 101 (2002).

${ }^{18}$ J. Long, Y. Qin, T. Nuhfer, M. De Graef, D. E. Lughlin, and M. E. McHenry, J. Appl. Phys. 101, 09N115 (2007). 